\title{
Preparation and characterization of calcium carbonate microspheres and their potential application as drug carriers
}

\author{
JUAN SONG, RAN WANG, ZAO LIU and HUASHAN ZHANG \\ School of Food and Biological Engineering, Hubei University of Technology, Wuhan, Hubei 430068, P.R. China
}

Received October 31, 2017; Accepted March 29, 2018

DOI: $10.3892 / \mathrm{mmr} .2018 .8879$

\begin{abstract}
The influence of lecithin from egg yolk (LE) on calcium carbonate $\left(\mathrm{CaCO}_{3}\right)$ biomineralization was investigated. In the present study, spherical $\mathrm{CaCO}_{3}$ particles were synthesized via coprecipitation in the presence of LE. LE multilamellar liposomes were first tuned by sonication to provide better control over the nucleation of $\mathrm{CaCO}_{3}$. Subsequently, monodisperse microspheres $\sim 2 \mu \mathrm{m}$ in size were generated by controlling the aggregation and growth of $\mathrm{CaCO}_{3}$ under appropriate concentrations of LE. In contrast to unstable vaterite, the microspheres generated in aqueous solution remained stable for at least 10 days without transforming into calcite, due to the strong interaction between the LE and calcium ions. The microspheres as drug carriers of doxorubicin (DOX) were assessed and were observed to have a good encapsulation efficiency, sustained drug release without a burst release and notable $\mathrm{pH}$ sensitivity. In addition, in vivo tumor inhibition examination demonstrated that DOX-loaded $\mathrm{CaCO}_{3}$ microspheres formulation had more superior efficacy to significantly restrain tumor growth. These novel $\mathrm{LE} / \mathrm{CaCO}_{3}$ hybrids may provide novel options for various biomedical applications.
\end{abstract}

\section{Introduction}

Lecithin from egg yolk (LE) is one of the best sources of phosphatides, of which the major components are phosphatidylcholine (PC) and sphingomyelin (SM). Its PC content is approximately $70-80 \%$. Its SM content is approximately $15-20 \%$ (1). Lecithin is a natural emulgator and a nutritious supplement. The functionalities and features of lecithin as well as its development and utilization have been receiving increasing attention from scientists worldwide (2-5). Actual

Correspondence to: Dr Juan Song, School of Food and Biological Engineering, Hubei University of Technology, 28 Nanli Road, Hongshan, Wuhan, Hubei 430068, P.R. China

E-mail: songjuan09@126.com

Key words: egg yolk, vaterite, biomaterials, $\mathrm{pH}$ sensitivity, drug release production process and clinical applications proved that LE was superior to other emulsifiers for intravenous injection, such as soya bean lecithin (6). Calcium carbonate $\left(\mathrm{CaCO}_{3}\right)$ is one of the minerals that exists naturally in abundance and is the major component of the shells of shellfish. It has been extensively applied in scientific and industrial fields (7-11). Biomimetic synthesis is a method of simulating biomineralization processes regulated by organic media to synthesize inorganic materials with complicated structures, highly ordered arrangements and special functionalities (12). The research on $\mathrm{CaCO}_{3}$ drug carriers mainly investigates the methods of producing a biomineral with favorable biological properties via biomimetic synthesis. Compared with other drug carriers, $\mathrm{CaCO}_{3}$ as a drug carrier has a series of merits such as a simple preparation process, sensitivity to $\mathrm{pH}$, and favorable biocompatibility and degradability in addition to being environmentally friendly and non-toxic to humans (13). The $\mathrm{pH}$ sensitivity of it could be favorable for the release of drugs because of the acidity of the lysosomes in cancer cells and the weak acid environment outside of cancer cells (14). Therefore, the $\mathrm{CaCO}_{3}$ would be an ideal drug carrier. At present, the anticancer effects of chemotherapy drugs are good in vitro but not ideal in vivo, the reasons of which might be the dosage form, administration method and microenvironment inside the body. Therefore, a change in the dosage form could increase the utilization of drugs to some extent. The two ends of phosphatide molecules are a negatively charged polar acyl group and a positively charged quaternary ammonium group, i.e., a non-polar hydrophobic end and a polar hydrophilic end. $\mathrm{LE}$ and $\mathrm{CaCO}_{3}$ both have favorable biocompatibility, degradability, and non-toxicity, among other features. This work investigated the regulation of LE-ordered systems on $\mathrm{CaCO}_{3}$ crystallization to prepare compound microparticles of $\mathrm{CaCO}_{3} / \mathrm{LE}$ and to realize the controllable release of anticancer drugs.

\section{Materials and methods}

Ethical statement. All animal experiments were performed in strict accordance with the recommendations in the Guide for the Care and Use of Laboratory Animals of the National Institutes of Health (Bethesda, MD, USA). The animal protocols were approved by the Committee on the Ethics of Animal Experiments of the Hubei University of Technology (Wuhan, China). 
Reagents and animals. Calcium chloride $\left(\mathrm{CaCl}_{2}\right)$ and sodium carbonate $\left(\mathrm{Na}_{2} \mathrm{CO}_{3}\right.$; Sinopharm Chemical Reagent Co., Ltd., Shanghai, China) were of analytical grade and used without further purification. The deionized water used in all experiments was obtained from a Milli-Q system with a resistivity greater than $18.2 \mathrm{M} \Omega \mathrm{cm}$. Doxorubicin hydrochloride (DOX) was purchased from Shanghai Macklin Biochemical Co., Ltd. (Shanghai, China). All glass was cleaned and sonicated in ethanol for $10 \mathrm{~min}$, soaked in a $\mathrm{H}_{2} \mathrm{O}-\mathrm{HNO}_{3}(65 \%)-\mathrm{H}_{2} \mathrm{O}_{2}$ (1:1:1 by volume) solution, rinsed with deionized water and acetone, and then dried in air. H22 hepatoma cells lines (purchased from the China center for type culture collection), Male Kunming (KM) mice (age, 6-12 wk, weight, 20 \pm 2 g) were purchased from the Hubei provincial academy of preventive medicine.

Polarized light microscopy (PLM) investigation of the LE ordered systems. PLM (Leica DM4500P; Leica Microsystems GmbH, Wetzlar, Germany) was employed to characterize the LE ordered systems. The sample for PLM investigation was prepared by dropping one drop of the LE-ordered system suspension onto a slide and placing a glass cover on top. The LE ordered systems were prepared by reconstituting LE in deionized water. Then, the sample was put under PLM for observation. The PLM image was recorded by a digital camera.

Preparation of $\mathrm{CaCO}_{3}$ in $\mathrm{LE}$. LE with different concentrations of $\mathrm{Ca}^{2+}$ was prepared by reconstituting $0,20,100$ and $250 \mathrm{mg}$ of $\mathrm{LE}$ in $10 \mathrm{ml}$ of a $0.3 \mathrm{~mol} / 1 \mathrm{CaCl}_{2}$ solution by sonication, respectively. The precipitation reaction was conducted by mixing $10 \mathrm{ml}$ of a $0.3 \mathrm{~mol} / 1 \mathrm{Na}_{2} \mathrm{CO}_{3}$ solution with the $\mathrm{Ca}^{2+} / \mathrm{LE}$ ordered systems by stirring. The precipitate formed immediately and was aged for $2 \mathrm{~h}$ before being rinsed with ethanol by centrifugation several times and dried for SEM and TEM analysis. Because the aging time of each sample was the same, it should not have any influence on the differences in the morphology and polymorph of the $\mathrm{CaCO}_{3}$ produced in the different reaction suspensions. A control sample of $\mathrm{CaCO}_{3}$ prepared without LE was produced by mixing $10 \mathrm{ml}$ of a $0.3 \mathrm{~mol} / 1 \mathrm{CaCl}_{2}$ solution with $10 \mathrm{ml}$ of a $0.3 \mathrm{~mol} / 1 \mathrm{Na}_{2} \mathrm{CO}_{3}$ solution by stirring and the precipitate was aged for $2 \mathrm{~h}$ as well. The concentrations of the $\mathrm{CaCl}_{2}$ and $\mathrm{Na}_{2} \mathrm{CO}_{3}$ solutions used in synthesizing the $\mathrm{CaCO}_{3}$ precipitation for XRD analysis were both $0.3 \mathrm{~mol} / \mathrm{l}$. The precipitation was also conducted in 5 , 10 and $15 \mathrm{mg} / \mathrm{ml} \mathrm{LE}$, respectively.

Loading and release of $\mathrm{DOX}$ in the $\mathrm{CaCO}_{3}$ microspheres. First, $20 \mathrm{mg}$ of $\mathrm{CaCO}_{3}$ microspheres was added into $2 \mathrm{ml}$ of an aqueous DOX solution $(0.5 \mathrm{mg} / \mathrm{ml})$. The mixture was shaken in a thermostatic shaker for $24 \mathrm{~h}$ at $25^{\circ} \mathrm{C}$. Then, the products were collected by centrifugation at $6,000 \mathrm{rpm}$ for $10 \mathrm{~min}$, washed twice with $1 \mathrm{ml}$ of deionized water, and dried at room temperature for future use. The DOX content of the supernatant was determined by measuring the absorbance at $481 \mathrm{~nm}$ with a Microplate spectrophotometer (Epoch2, BioTek, VT, USA) against a calibration curve. The loading content and entrapment efficiency of DOX was calculated as follows: Drug loading content=(WD-WF $) / \mathrm{WCx} 100 \%$; and Entrapment efficiency $=(\mathrm{WD}-\mathrm{WF}) / \mathrm{WD} \times 100 \%$.

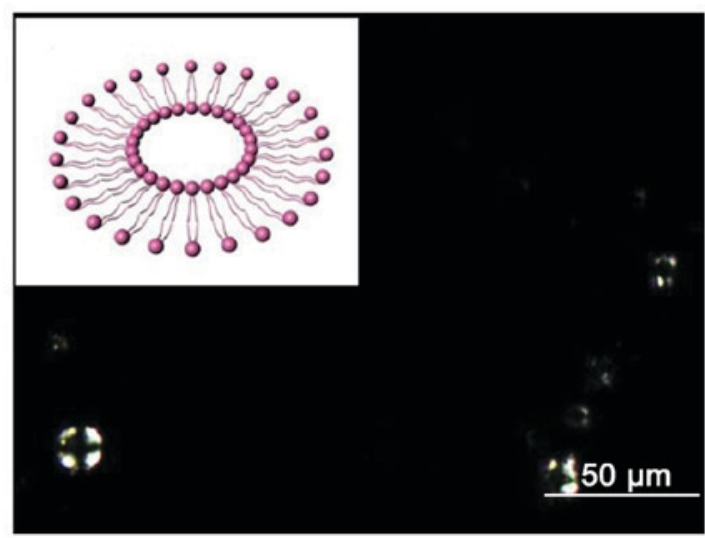

Figure 1. Polarized light microscopy image of the lecithin- $\mathrm{H}_{2} \mathrm{O}$ system structure. The inset image illustrates the structure of a multilamellar liposome. Scale bar, $50 \mu \mathrm{m}$.

where WD is the total weight of DOX in the reaction, WF is the total weight of free DOX remaining in the supernatant, and WC is the total weight of the $\mathrm{CaCO}_{3}$ microspheres loaded with DOX.

For the drug release studies, $20 \mathrm{mg}$ of the dried DOX-loaded $\mathrm{CaCO}_{3}$ microspheres (DCM) after the washing treatment was added into $2 \mathrm{ml}$ of PBS solutions at different $\mathrm{pH}$ values (4, 6.5 and 7.4 ) and placed in a thermostatic shaker at $37^{\circ} \mathrm{C}$. At predetermined intervals, $1 \mathrm{ml}$ of the supernatant was taken and analyzed by measuring the absorbance at $481 \mathrm{~nm}$. The same volume $(1 \mathrm{ml})$ of fresh PBS buffer at different $\mathrm{pH}$ values was added into the release medium. The release behaviors vs. time were evaluated based on one sample, and the release measurement was performed in triplicate.

Characterization. The morphologies of the $\mathrm{CaCO}_{3}$ samples were characterized with scanning electron microscopy (SEM, JSM6390LV, JEOL, Ltd., Tokyo, Japan; S-4700; Cold Field Emission SEM, Hitachi, Ltd., Tokyo, Japan). The samples were sputter-coated with platinum before being measured with SEM. Transmission electron microscopy (TEM, JEM-2100; JEOL, Ltd.) was used to investigate the morphology and microstructure of the precipitation. The content of LE in the microspheres was measured through thermogravimetric analysis (TGA; Diamond TG/DTA; PerkinElmer, Inc., Waltham, MA, USA) at $10^{\circ} \mathrm{C} \mathrm{m^{-1 }}$ in nitrogen gas with a flow rate of $40 \mathrm{~cm}^{3} \mathrm{~min}^{-1}$. X-ray diffraction (XRD; X'pert Powder; PANalytical BV, Almelo, The Netherlands) was employed to determine the polymorphs of $\mathrm{CaCO}_{3}$ precipitation. The operating conditions for the XRD were as follows: Radiation of $\mathrm{CuK} \alpha$, tube voltage of $40 \mathrm{kV}$, tube current of $30 \mathrm{~mA}$, scanning rate of $0.6 \% \mathrm{~min}$ and a collecting angle (20) ranging from $10^{\circ}$ to $60^{\circ}$. Fluorescence images of the DCM were captured by a fluorescence inverted microscope (Axio Vert A1; Zeiss AG, Oberkochen, Germany).

In vivo antitumor effect. Mice were maintained at $25 \pm 1^{\circ} \mathrm{C}$ and $60 \pm 5 \%$ humidity under a $12 \mathrm{~h}$ light-dark cycle. All experimental animals were housed under specific-pathogen-free conditions for 1 week to get accustomed to the surroundings before initiation of the experiment. The model of the tumor-bearing mice was created by subcutaneous injection of $\mathrm{H} 22$ cells as previously described (15-17). Briefly, the H22 

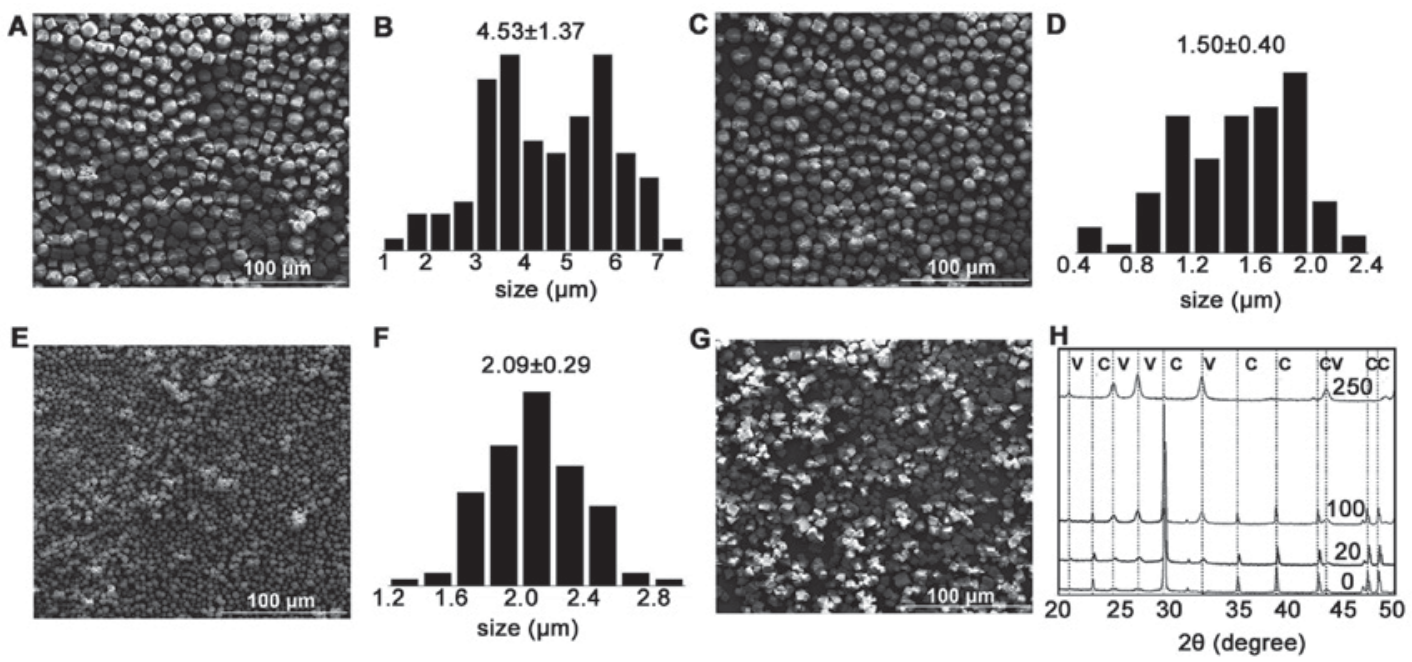

Figure 2. (A-G) Scanning electron microscopy images and (H) X-ray diffraction patterns of $\mathrm{CaCO}_{3}$ particles obtained under a constant Ca ion concentration $(0.3 \mathrm{~mol} / \mathrm{l})$ and various lecithin concentrations. (A and B) $20 \mathrm{mg}$, (C and D) $100 \mathrm{mg}$, (E and F) $250 \mathrm{mg}$ and (G) $0 \mathrm{mg}$. (B, D and F) Present the size distribution of the relative $\mathrm{CaCO}_{3}$ particles. Scale bars, $100 \mu \mathrm{m} . \mathrm{CaCO}_{3}$, calcium carbonate.

cells with ascites were harvested, diluted to a concentration of $5.0 \times 10^{6} / \mathrm{ml}$ with sterilized normal saline (NS), and inoculated subcutaneously into the right armpit region of the mice. The mice were divided into 3 group with 10 mice in each group, on seventh day of inoculated treatments. The mice in the control group were treated with NS. The animals in the other 2 groups were admisistered DOS group or DCM group, through the tail vein at a dose of $5 \mathrm{mg} / \mathrm{kg}$ DOX for the single dose. After tail vein injection for 7 days, the mice were killed by pulling and breaking of the cervical vertebra. The tumors were excised and weighted. The inhibitory effect of experimental treatment on tumor growth was evaluated by tumor inhibition rate. The inhibitory rates of tumor growth were calculated as follows $(17,18)$ : Tumor inhibition rate $=(\mathrm{Wc}-\mathrm{Ws}) / \mathrm{Wcx} 100 \%$, where $\mathrm{Wc}$ and $\mathrm{Ws}$ were donated as the tumor weight in the control and sample groups, respectively.

Statistical analyses. All experiments were performed at least three times and expressed as mean \pm standard deviation (SD). Data were analyzed for statistical significance using SPSS 14.0 (SPSS, Inc., Chicago, IL, USA), and analysis of variance and the Student-Newman-Keuls post hoc test were performed for multiple comparisons. $\mathrm{P}<0.05$ was considered to indicate a statistically significant difference.

\section{Results}

The structure of the $\mathrm{LE}-\mathrm{H}_{2} \mathrm{O}$ system investigated by PLM. As shown in Fig. 1, there were many Maltese crosses in the LE- $\mathrm{H}_{2} \mathrm{O}$ structure under cross-polarized light.

Effect of $\mathrm{LE}$ on the morphology of $\mathrm{CaCO}_{3}$ particles. The SEM images shown in Fig. 2 represent the morphology of the $\mathrm{CaCO}_{3}$ precipitation from the reconstitution of $0,20,100$ and $250 \mathrm{mg}$ of LE. The precipitation of the control sample, $0 \mathrm{mg}$ of LE, showed an irregular cubic shape of the particles, which is quite common for $\mathrm{CaCO}_{3}$. In the precipitation of $20 \mathrm{mg}$ of $\mathrm{LE}$, most of the particles were uniform cubic crystals with lamellar structure; the lecithin concentration was less than the critical
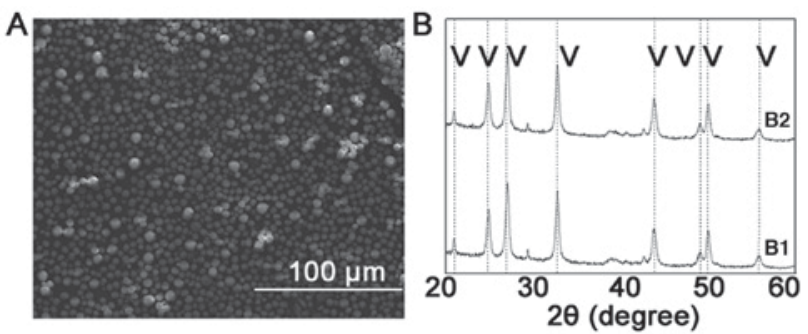

Figure 3. Stability of the microspheres. (A) Scanning electron microscopy image of $\mathrm{CaCO}_{3}$ particles containing lecithin following incubation for 10 days in a flowing water solution. (B) X-ray diffraction patterns of the $\mathrm{CaCO}_{3}$ particles prior to $\mathrm{B} 1$ and following B). Scale bar, $100 \mu \mathrm{m} . \mathrm{CaCO}_{3}$, calcium carbonate.

micelle concentration $(\mathrm{CMC}=1.6 \mathrm{mmol} / \mathrm{l})$. In $100 \mathrm{mg} \mathrm{LE}$, the particles were uniform cubic crystals and, with the exception of some spherical particles, the amount of rough spherical particles increased. In $250 \mathrm{mg} \mathrm{LE}$, spherical particles dominated the precipitation; the spherical particles exhibited fine homogeneity and a porous structure.

Stability of the $\mathrm{CaCO}_{3}$ microspheres. The formation and stabilization of $\mathrm{CaCO}_{3}$ particles remain fundamental parameters to their unique properties $(19,20)$. Therefore, the LE-regulated microspheres were incubated in a flowing water solution to assess their stability. All typical peaks remained, and no calcite peak appeared by XRD after the water flow process (Fig. 3), confirming the stability of the LE-regulated microspheres.

$\mathrm{CaCO}_{3}$ microspheres formation process. It was confirmed that the $\mathrm{LE}$ used as the organic template for $\mathrm{CaCO}_{3}$ precipitation consisted of vesicles with multilamellar structure. This indicated that the microenvironment in the LE suspension increasingly favored the formation of aggregated balls. Fig. 4 shows the morphology of the porous balls formed as aggregates of the nanoparticles. To investigate the structure of the LE precipitation, HRTEM and selected area electron 
Table I. Inhibitory effect of DCM on the growth of $\mathrm{H} 22$ tumors transplanted in mice.

\begin{tabular}{lcc}
\hline Group & Tumor weight $(\mathrm{g})$ & Inhibition rate $(\%)$ \\
\hline NS & $2.04 \pm 0.67$ & - \\
DOS & $1.28 \pm 0.28^{\mathrm{a}}$ & 37.30 \\
DCM & $0.73 \pm 0.19^{\mathrm{b}, \mathrm{c}}$ & $64.23^{\mathrm{c}}$ \\
\hline
\end{tabular}

Data are expressed as mean \pm standard deviation $(n=10)$. ${ }^{\mathrm{a}} \mathrm{P}<0.05$ and ${ }^{\mathrm{b}} \mathrm{P}<0.01$ vs. NS group; ${ }^{\mathrm{c}} \mathrm{P}<0.01$ vs. DOX group. NS, normal saline; DCM, DOX-loaded $\mathrm{CaCO}_{3}$ microspheres; DOX, doxorubicin; $\mathrm{CaCO}_{3}$, calcium carbonate.
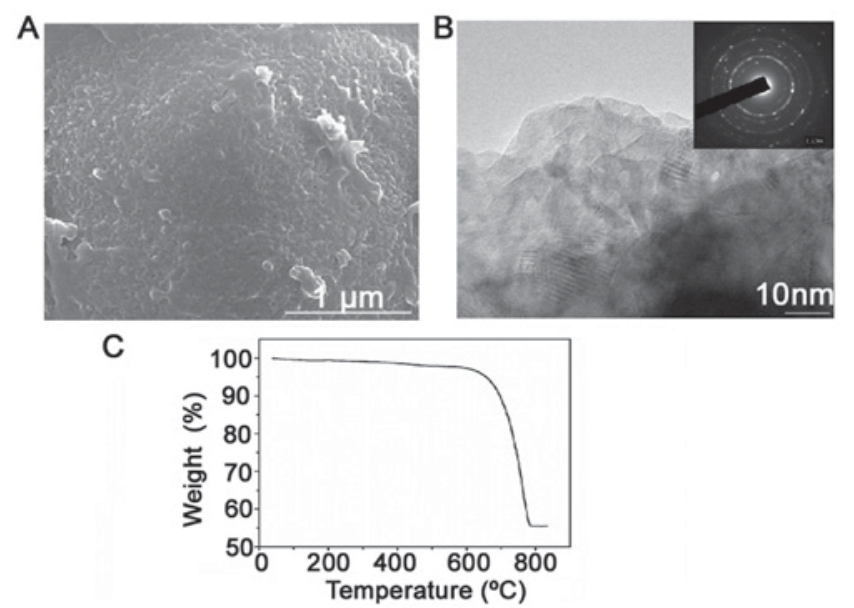

Figure 4. Microsphere structure and the lecithin distribution in the microsphere. (A) Scanning electron microscopy image and element mapping. Scale bar, $1 \mu \mathrm{m}$ (B) Transmission electron microscopy image and selected area electron diffraction pattern. Scale bar, $10 \mathrm{~nm}$. (C) Thermogravimetric analysis curve.

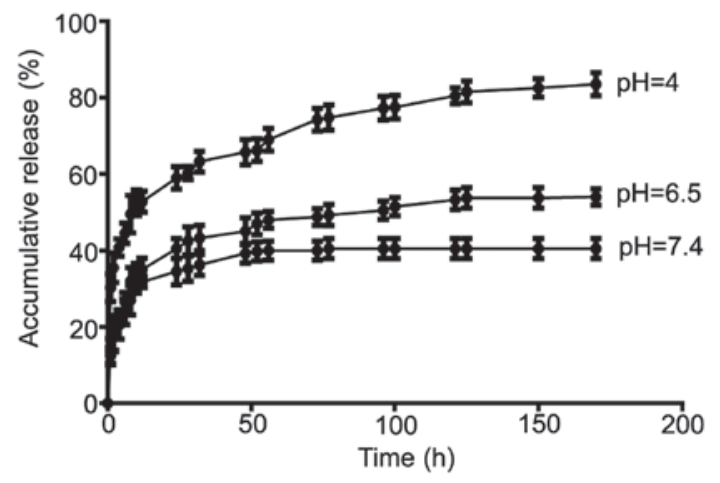

Figure 5. In vitro drug release profiles of doxorubicin-loaded $\mathrm{CaCO}_{3}$ microspheres in different $\mathrm{pH}$ media. $\mathrm{CaCO}_{3}$, calcium carbonate.

diffraction (SAED) were employed. It revealed the approximate size $(50 \mathrm{~nm})$ and morphology of the nanoparticles. The nanoparticles, which aggregated and formed the hierarchical structure of the $\mathrm{CaCO}_{3}$ particles, were more obvious. The $\mathrm{CaCO}_{3}$ spherical particles that appeared were formed and aggregated by numerous nanosized subparticles. TGA and elemental mapping revealed the content and distribution of LE in the microspheres (Fig. 4C). During TGA, weight loss from
200 to $600^{\circ} \mathrm{C}$ was observed, with only a small amount ascribed to the degradation of the lecithin.

Drug loading and release from LE-regulated microspheres. The drug loading and release behavior of the microspheres were assessed with DOX as a model drug. Absorbance analysis showed that the drug loading content and the entrapment efficiency of DOX was $5.1 \pm 0.1$ and $82.5 \pm 3.1 \%$, respectively, when the ratio of the drug to microspheres was 1:20 (w/w). The in vitro release of DOX from the vaterite microspheres at $\mathrm{pH}$ 7.4, 6.5 and 4 is shown in Fig. 5. The DOX release from the microspheres was also strongly $\mathrm{pH}$ dependenthighest at a $\mathrm{pH}$ of 4 , then at a $\mathrm{pH}$ of 6.5 , lowest at a $\mathrm{pH}$ of 7.4. After 8 days, the amount of DOX released at $\mathrm{pH} 7.4$ was $40 \%$ of the total drug load but reached $80 \%$ at a $\mathrm{pH}$ of 4 . At $\mathrm{pH} 6.5$, the release of DOX was triggered through the initial $38 \mathrm{~h}$ only about 40-50\% DOX release, and continued at a slower but steady pace.

Tumor inhibition rates. After mice were killed, the solid tumors were removed from tumor-bearing mice and the tumor inhibition rate was shown in Table I. Although the two DOX formulations both inhibited the growth of tumor compared with NS, the treatment of DCM showed a significantly better efficacy than that of free DOX. The average tumor weight in the DCM group was just half of that in the free DOX group $(\mathrm{P}<0.01)$. The tumor inhibition rates in $\mathrm{DCM}$ group was significantly higher than in the DOX group $(\mathrm{P}<0.01)$.

\section{Discussion}

The Maltese cross corresponded to a multilamellar vesicle structure. The PLM results proved that in the $\mathrm{LE}-\mathrm{H}_{2} \mathrm{O}$ system suspension, LE molecules self-assembled and packed into multilamellar liposome vesicles. Thus, it was confirmed that the LE liposomes used as the organic template for $\mathrm{CaCO}_{3}$ precipitation were vesicles with multilamellar structure.

The SEM results showed that as the concentration of LE increased, the amount of aggregated spherical particles in the precipitation increased. From the results and discussion above, it can be summarized that as the concentration of LE increased, the number of aggregated spherical particles in the precipitation increased accordingly. This indicates that the microenvironment in $\mathrm{LE}-\mathrm{H}_{2} \mathrm{O}$ increasingly favored the formation of aggregated balls. The interaction of LE and calcium ions was likely responsible for this result. LE induced a localized supersaturation zone of $\mathrm{Ca}^{2+}$, which remarkably increased the number of nuclei.

In the stability study, the $\mathrm{CaCO}_{3}$ microspheres were incubated in water after 10 days and retained their initial spherical morphology and size, suggesting the stability of those features. Here, in this research, it was suggested that in the LE suspension, the negatively charged phosphatidyl group can selectively bind $\mathrm{Ca}^{2+}$. When the LE formed in $\mathrm{Ca}^{2+}$ solution, the calcium ions were trapped and enriched by the binding sites on the negatively charged surface of the outermost bilayer of vesicles. Therefore, the outer surface of the LE vesicles acted as the template to modify the nucleation and growth of $\mathrm{CaCO}_{3}$. $\mathrm{LE}$ and $\mathrm{CaCO}_{3}$ must be closely combined to prevent the dissolution and recrystallization of the $\mathrm{CaCO}_{3}$. 


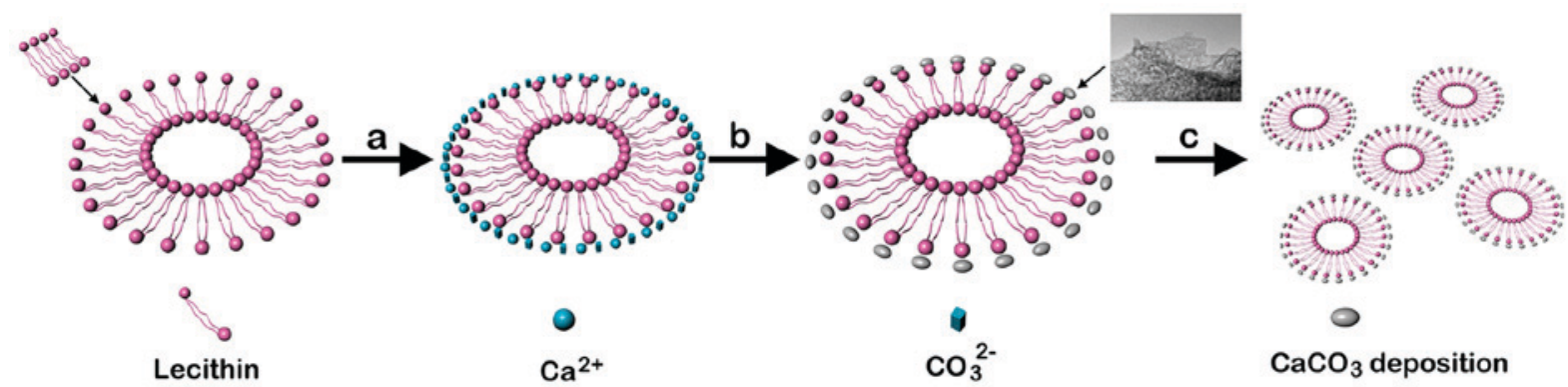

Figure 6. Schematic of the proposed formation process of the microspheres. $\mathrm{CaCO}_{3}$, calcium carbonate.

The $\mathrm{CaCO}_{3}$ microsphere formation process results suggest that the lecithin must be closely packed with $\mathrm{CaCO}_{3}$ precipitation to provide sufficient stabilization and prevent transformation. As shown in Fig. 6, the nucleation and formation of the nanoparticles in the $\mathrm{CaCO}_{3}$ precipitate was illustrated. The external additives adsorbed on these nanoparticles cause the nanoparticles to be attracted to each other, driving the directed self-assembly process. Eventually, the nanoparticles integrated with each other to form mesocrystals, and the additives existed between the particles. Therefore, the nanoparticle aggregation pathway is that which minimizes the surface energy and interfacial crystal energy, and this pathway is the directed accretion process. More significantly, the amphiphilic molecules attached to the microcrystal planes prevent the transformation.

The release of DOX from $\mathrm{CaCO}_{3}$ microsphere showed distinct $\mathrm{pH}$ dependence. The higher amount of drug release at $\mathrm{pH} 4.5$ could be caused by special structure of the $\mathrm{CaCO}_{3}$ microsphere based on ultra-pH-sensitivity, which was beneficial for rapid killing tumor cells. The amount of drug release at pH 6.5 was little high, but in vivo drug release might be less because of the increased acidity distribution from outer layer to the core of tumor tissue (21-23). Vaterite is a useful candidate for drug release due to its large surface area, biocompatibility, biodegradability, lower toxicity, low production costs, and $\mathrm{pH}$-dependent dissolution $(24,25)$. The vaterite particles with smaller and homogeneous sizes are preferred for drug delivery because of their improved efficient and homogeneous distribution of drugs as well as their improved cellular uptake $(26,27)$. Since the submicrometer $\mathrm{CaCO}_{3}$ carriers have larger specific surface areas, the increased drug entrapment efficiency of the microspheres in the present work may be due to the lecithin embedded in the particles, where the hydrophilic group could form strong interactions with DOX. Because of the stronger interactions of DOX and LE, an initial burst release, as in previous $\mathrm{CaCO}_{3}$-based release systems (28-34), was significantly reduced, resulting in sustained release for more than 1 week. The DOX release from the microspheres was also strongly $\mathrm{pH}$ sensitive. Compared with previous other microspheres (30), the slower release from vaterite microspheres suggested stronger interactions between DOX and microspheres.

In vivo tumor inhibition examination, the tumor treated with NS could not controlled and grew up rapidly. The tumor treated with free DOX and DCM was grew steadily. DCM was successful to a certain extent in controlling the tumor growth. Importantly, mice treated with DCM significantly reduced the tumor progression and displayed a remarkable tumor inhibition effect on tumor, which was possibly due to its enhanced drug accumulation and cell killing ability.

In conclusion, the results of the present study demonstrated that $\mathrm{CaCO}_{3}$ microspheres with smaller sizes can be prepared with LE as a modifier. The polymorphology and nanostructure of these microspheres were regulated by altering experimental parameters such as the LE concentration. The microspheres were stabilized by LE to prevent transformation into calcite. Higher DOX encapsulation and improved interactions supported sustained drug release and $\mathrm{pH}$-sensitive release behavior, suggesting the utility of the microspheres as drug carriers. These results provide new insight into the relationship between $\mathrm{LE}$ and $\mathrm{CaCO}_{3}$ biomineralization as well as a facile, efficient way to rapidly synthesize well-controlled $\mathrm{CaCO}_{3}$ particles. In addition, this DCM were found good anti-tumor effect in H22 tumor-bearing mice in vivo.

\section{Acknowledgements}

Not applicable.

\section{Funding}

No funding was received.

\section{Availability of data and materials}

All data generated or analyzed during this study are included in this published article.

\section{Authors' contributions}

JS designed experiments, and JS, RW and ZL carried out experiments. JS and $\mathrm{HZ}$ analyzed the experimental results, and wrote the manuscript. All authors read and approved the final manuscript.

\section{Ethics approval and consent to participate}

The animal protocols were approved by the Committee on the Ethics of Animal Experiments of the Hubei University of Technology (Wuhan, China).

\section{Consent for publication}

Not applicable. 


\section{Competing interests}

The authors declare that they have no competing interests.

\section{References}

1. Chi Y and Lin S: Research advances in the extraction and application of egg-yolk lecithin. Food Ferment Ind 28: 50-53, 2002.

2. Li Y, Liu JB and Lin SY: Study on extraction of high pure egg yolk lecithin. Food Sci 27: 851-853, 2006.

3. Palacios LE and Wang T: Extraction of egg-yolk lecithin. J Am Oil Chem Soc 82: 565-569, 2005.

4. Chang H, Wang EL, Gong XT and Liu JB: Over view on study of yolk lecithin. Sci Tech Food Ind 5: 414-420, 2010.

5. Ali AH, Zou X, Lu J, Abed SM, Yao Y, Tao G, Jin Q and Wang X: Identification of phospholipids classes and molecular species in different types of egg yolk by using UPLC-Q-TOF-MS. Food Chem 221: 58-66, 2017.

6. Rossi J and Leroux JC: Principles in the development of intravenous lipid emulsions. In: Wasan KM. Wiley (eds.) John Wiley \& Sons, Inc., New York, pp88-123, 2006.

7. Foran E, Weiner S and Fine M: Biogenic fish-gut calcium carbonate is a stable amorphous phase in the gilt-head seabream, sparus aurata. Sci Rep 3: 1700, 2013.

8. Vlieg E: Materials science. Complexity from simplicity. Science 340: 822-823, 2013.

9. Kim S and Park CB: Bio-inspired synthesis of minerals for energy, environment, and medicinal applications. Adv Funct Mater 23: 10-25, 2013.

10. Zhao Y, Luo Z, Li M, Qu Q, Ma X, Yu SH and Zhao Y: A preloaded amorphous calcium carbonate/doxorubicin@silica nanoreactor for $\mathrm{pH}$-responsive delivery of an anticancer drug. Angew Chem Int Ed Engl 54: 919-922, 2015.

11. Wang C, Chen SQ, Yu Q, Hu FQ and Yuan H: Taking advantage of the disadvantage: Employing the high aqueous instability of amorphous calcium carbonate to realize burst drug release within cancer cells. J Mater Chem B 5: 2068-2073, 2017.

12. Zan G and Wu Q: Biomimetic and bioinspired synthesis of nanomaterials/nanostructures. Adv Mater 28: 2099-2147, 2016.

13. Han MR, Kwon MC, Lee HY, Kim JC, Kim JD, Yoo SK, SIN IS and Kim SM: pH-dependent release property of alginate beads containing calcium carbonate particles. J Microencapsul 24: 787-796, 2007.

14. Wei W, Ma GH, Hu G, Yu D, Mcleish T, Su ZG and Shen ZY: Preparation of hierarchical hollow $\mathrm{CaCO}_{3}$ Particles and the application as anticancer drug carrier. J Am Chem Soc 130: 15808-15810, 2008.

15. Xiaoguang C, Hongyan L, Xiaohong L, Zhaodi F, Yan L, Lihua T and Rui H: Cancer chemopreventive and therapeutic activities of red ginseng. J Ethnopharmacol 60: 71-78, 1998.

16. Gao L, Chen L, Fei XH, Qiu HY, Zhou H and Wang JM: STI571 combined with vincristine greatly suppressed the tumor formation of multidrug-resistant K562 cells in a human-nude mice xenograft model. Chin Med J (Engl) 119: 911-918, 2006.

17. Jin Y, Li J, Rong LF, Li YH, Guo L and Xu SY: Anti-hepatocarcinoma effects of 5-fluorouracil encapsulated by galactosylceramide liposomes in vivo and in vitro. World J Gastroenterol 11: 2643-2646, 2005.

18. Chen H, Takahashi S, Imamura M, Okutani E, Zhang ZG, Chayama $\mathrm{K}$ and Chen BA: Earthworm fibrinolytic enzyme: Anti-tumor activity on human hepatoma cells in vitro and in vivo. Chin Med J (Engl) 120: 898-904, 2007.
19. Naka K, Huang SC and Chujo Y: Formation of stable vaterite with poly(acrylic acid) by the delayed addition method. Langmuir 22: 7760-7767, 2006

20. Naka K, Tanaka Y and Chujo Y: Effect of anionic starburst dendrimers on the crystallization of $\mathrm{CaCO}_{3}$ in aqueous solution: Size control of spherical vaterite particles. Langmuir 18: 3655-3658, 2002

21. Helmlinger G, Yuan F, Dellian M, Dellian M and Jain RK: Interstitial $\mathrm{pH}$ and $\mathrm{pO} 2$ gradients in solid tumors in vivo: High-resolution measurements reveal a lack of correlation. Nat Med 3: 177-182, 1997.

22. Hirschhaeuser F, Menne H, Dittfeld C, West J, Mueller-Klieser W and Kunz-Schughart LA: Multicellular tumor spheroids: An underestimated tool is catching up again. J Biotechnol 148: 3-15, 2010.

23. Chauhan VP and Jain RK: Strategies for advancing cancer nanomedicine. Nat Mater 12: 958-962, 2013.

24. Parakhonskiy BV, Haase A and Antolini R: Sub-micrometer vaterite containers: Synthesis, substance loading, and release. Angew Chem Int Ed Engl 51: 1195-1197, 2012.

25. Schmidt $\mathrm{S}$ and Volodkin D: Microparticulate biomolecules by mild $\mathrm{CaCO}_{3}$ templating. J Mater Chem B 1: 1210-1218, 2013.

26. Qi C, Zhu YJ and Chen F: Microwave hydrothermal transformation of amorphous calcium carbonate nanospheres and application in protein adsorption. ACS Appl Mater Interfaces 6: 4310-4320, 2014.

27. Svenskaya Y, Parakhonskiy B, Haase A, Atkin V, Lukyanets E, Gorin D and Antolini R: Anticancer drug delivery system based on calcium carbonate particles loaded with a photosensitizer. Biophys Chem 182: 11-15, 2013.

28. Yang YH, Liu CH, Liang YH, Lin FH and Wu KCW: Hollow mesoporous hydroxyapatite nanoparticles (hmHANPs) with enhanced drug loading and $\mathrm{PH}-\mathrm{responsive} \mathrm{release} \mathrm{properties}$ for intracellular drug delivery. J Mater Chem B 1: 2447-2450, 2013.

29. Qi C, Zhu YJ and Chen F: Microwave hydrothermal transformation of amorphous calcium carbonate nanospheres and application in protein adsorption. ACS Appl Mater Interfaces 6: 4310-4320, 2014

30. Wang J, Chen JS, Zong JY, Zhao D, Li F, Zhou RX and Cheng SX: Calcium carbonate/carboxymethyl chitosan hybrid microspheres and nanospheres for drug delivery. J Phys Chem C 114: 18940-18945, 2010.

31. Ying $X$, Shan $C$, Jiang $K$, Chen $Z$ and Du Y: Intracellular pH-sensitive delivery $\mathrm{CaCO}_{3}$ nanoparticles templated by hydrophobic modified starch micelles. RSC Adv 4: 10841-10844, 2014.

32. Kurapati R and Raichur AM: Composite cyclodextrin-calcium carbonate porous microparticles and modified multilayer capsules: Novel carriers for encapsulation of hydrophobic drugs. J Mater Chem B 1: 3175-3184, 2013

33. Peng C, Zhao Q and Gao C: Sustained delivery of doxorubicin by porous $\mathrm{CaCO}_{3}$ and chitosan/alginate multilayers-coated $\mathrm{CaCO}_{3}$ microparticles. Colloids Sur A: Physicochem Eng Aspects 353: 132-139, 2010.

34. Parakhonskiy BV, Foss C, Carletti E, Fedel M, Haase A, Motta A, Migliaresi $\mathrm{C}$ and Antolini R: Tailored intracellular delivery via a crystal phase transition in $400 \mathrm{~nm}$ vaterite particles. Biomater Sci 1: 1273-1281, 2013. 